TAIWANESE JOURNAL OF MATHEMATICS

Vol. 10, No. 3, pp. 669-690, March 2006

This paper is available online at http://www.math.nthu.edu.tw/tjm/

\title{
WEIGHTED BOUNDEDNESS OF MULTILINEAR OPERATORS FOR THE EXTREME CASES
}

\author{
Liu Lanzhe
}

\begin{abstract}
In this paper, the weighted boundedness of multilinear operators related to some non-convolution operators for the extreme cases are obtained. The operators include Littlewood-Paley operator, Marcinkiewicz operator and Bochner-Riesz operator.
\end{abstract}

\section{INTRODUCTION}

Let $T$ be a Calderon-Zygmund operator, a classical result of Coifman, Rochberg and Weiss (see [8]) states that the commutator $[b, T]=b T f-T(b f)$ (where $b \in$ $B M O\left(R^{n}\right)$ ) is bounded on $L^{p}\left(R^{n}\right)$ for $1<p<\infty$; Chanillo (see [2]) proved a similar result when $T$ is replaced by the fractional integral operator. In [11], the endpoint boundedness of the commutators are obtained. The main purpose of this paper is to discuss the weighted endpoint boundedness of multilinear operators related to some non-convolution operators. In fact, we shall establish the weighted boundedness in the extreme cases of $p$ for the multilinear operators related to some non-convolution operator only under certain size conditions of the operators. As application, the weighted endpoint boundedness of the multilinear operators related to the Littlewood-Paley operator, Marcinkiewicz operator and Bochner-Riesz operator are obtained.

\section{Preliminaries}

Throughout this paper, $Q$ will denote a cube of $R^{n}$ with sides parallel to the axes. For a cube $Q$ and a locally integrable function $f$, let $f(Q)=\int_{Q} f(x) d x$,

Received September 26, 2003; revised March 5, 2004.

Communicated by Sen-Yen Shaw.

2000 Mathematics Subject Classification: 42B20, 42B25.

Key words and phrases: Multilinear operator, Littlewood-Paley operator, Marcinkiewicz operator, Bochner-Riesz operator, BMO space, Hardy space.

Supported by the NNSF(Grant: 10271071 . 
$f_{Q}=|Q|^{-1} \int_{Q} f(x) d x$ and $f^{\#}(x)=\sup _{x \in Q}|Q|^{-1} \int_{Q}\left|f(y)-f_{Q}\right| d y$. Moreover, for a weight function $w, f$ is said to belong to $B M O(w)$ if $f^{\#} \in L^{\infty}(w)$ and define $\|f\|_{B M O(w)}=\left\|f^{\#}\right\|_{L^{\infty}(w)}$, if $w=1$, we denote that $B M O(w)=\operatorname{BMO}\left(R^{n}\right)$. Also, we give the concepts of the atom and weighted $H^{1}$ space. A function $a$ is called a $H^{1}$ atom if there exists a cube $Q$ such that $a$ is supported on $Q,\|a\|_{L^{\infty}(w)} \leq$ $w(Q)^{-1}$ and $\int a(x) d x=0$. It is well known that the weighted Hardy space $H^{1}(w)$ has the atomic decomposition characterization(see[1] [10]).

In this paper, we will consider a class of multilinear operators related to some integral operators as follows.

Let $m$ be a positive integer and $A$ be a function on $R^{n}$. We denote

$$
R_{m+1}(A ; x, y)=A(x)-\sum_{|\alpha| \leq m} \frac{1}{\alpha !}(x-y)^{\alpha} D^{\alpha} A(y)
$$

and

$$
Q_{m+1}(A ; x, y)=R_{m}(A ; x, y)-\sum_{|\alpha|=m}(x-y)^{\alpha} D^{\alpha} A(x) .
$$

Definition 1. Let $F(x, y, t)$ be a function defined on $R^{n} \times R^{n} \times[0,+\infty)$, and let

$$
F_{t}(f)(x)=\int_{R^{n}} F(x, y, t) f(y) d y
$$

and

$$
F_{t}^{A}(f)(x)=\int_{R^{n}} \frac{R_{m+1}(A ; x, y)}{|x-y|^{m}} F(x, y, t) f(y) d y .
$$

Let $H$ be a Banach space $H=\{h:\|h\|<\infty\}$ such that $F_{t}(f)(x)$ and $F_{t}^{A}(f)(x)$ may be viewed as a mapping from $[0,+\infty)$ to $H$ for each fixed $x \in R^{n}$. Then, the multilinear operators related to $F_{t}$ is defined by

$$
T^{A}(f)(x)=\left\|F_{t}^{A}(f)(x)\right\| .
$$

We also define that $T(f)(x)=\left\|F_{t}(f)(x)\right\|$.

In particular, we shall study the following sublinear operators.

Definition 2. Let $\varepsilon>0$ and $\psi$ be a fixed function which satisfies the following properties:

(1) $\int_{R^{n}} \psi(x) d x=0$

(2) $|\psi(x)| \leq C(1+|x|)^{-(n+1)}$,

(3) $|\psi(x+y)-\psi(x)| \leq C|y|^{\varepsilon}(1+|x|)^{-(n+1+\varepsilon)}$ for $2|y|<|x|$. 
The multilinear Littlewood-Paley operator is defined by

$$
g_{\psi}^{A}(f)(x)=\left(\int_{0}^{\infty}\left|F_{t}^{A}(f)(x)\right|^{2} \frac{d t}{t}\right)^{1 / 2},
$$

where

$$
F_{t}^{A}(f)(x)=\int_{R^{n}} \frac{R_{m+1}(A ; x, y)}{|x-y|^{m}} \psi_{t}(x-y) f(y) d y,
$$

and $\psi_{t}(x)=t^{-n} \psi(x / t)$ for $t>0$. Let $F_{t}(f)=\psi_{t} * f$. We also define

$$
g_{\psi}(f)(x)=\left(\int_{0}^{\infty}\left|F_{t}(f)(x)\right|^{2} \frac{d t}{t}\right)^{1 / 2},
$$

which is the Littlewood-Paley $g$ function (see [17]).

We also consider the variant of $g_{\psi}^{A}$, which is defined by

$$
\tilde{g}_{\psi}^{A}(f)(x)=\left(\int_{0}^{\infty}\left|\tilde{F}_{t}^{A}(f)(x)\right|^{2} \frac{d t}{t}\right)^{1 / 2}
$$

where

$$
\tilde{F}_{t}^{A}(f)(x)=\int_{R^{n}} \frac{Q_{m+1}(A ; x, y)}{|x-y|^{m}} \psi_{t}(x-y) f(y) d y .
$$

Definition 3. Let $0<\gamma \leq 1$ and $\Omega$ be homogeneous of degree zero on $R^{n}$ such that $\int_{S^{n-1}} \Omega\left(x^{\prime}\right) d \sigma\left(x^{\prime}\right)=0$. Assume that $\Omega \in \operatorname{Lip}_{\gamma}\left(S^{n-1}\right)$, that is, there exists a constant $M>0$ such that for any $x, y \in S^{n-1},|\Omega(x)-\Omega(y)| \leq M|x-y|^{\gamma}$.

We denote $\Gamma(x)=\left\{(y, t) \in R_{+}^{n+1}:|x-y|<t\right\}$ and the characteristic of $\Gamma(x)$ by $\chi_{\Gamma(x)}$. The multilinear Marcinkiewicz operator is defined by

$$
\mu_{\Omega}^{A}(f)(x)=\left[\int_{0}^{\infty}\left|F_{t}^{A}(f)(x)\right|^{2} \frac{d t}{t^{3}}\right]^{1 / 2},
$$

where

$$
F_{t}^{A}(f)(x)=\int_{|x-y| \leq t} \frac{\Omega(x-y)}{|x-y|^{n-1}} \frac{R_{m+1}(A ; x, y)}{|x-y|^{m}} f(y) d y
$$

Let

$$
F_{t}(f)(x)=\int_{|x-y| \leq t} \frac{\Omega(x-y)}{|x-y|^{n-1}} f(y) d y .
$$

We also define that

$$
\mu_{\Omega}(f)(x)=\left(\int_{0}^{\infty}\left|F_{t}(f)(x)\right|^{2} \frac{d t}{t^{3}}\right)^{1 / 2}
$$


where the last is the Marcinkiewicz operator (see [18]). Also, the variant of $\mu_{\Omega}^{A}$ is defined by

$$
\tilde{\mu}_{\Omega}^{A}(f)(x)=\left(\int_{0}^{\infty}\left|\tilde{F}_{t}^{A}(f)(x)\right|^{2} \frac{d t}{t^{3}}\right)^{1 / 2},
$$

where

$$
\tilde{F}_{t}^{A}(f)(x)=\int_{|x-y| \leq t} \frac{\Omega(x-y)}{|x-y|^{n-1}} \frac{Q_{m+1}(A ; x, y)}{|x-y|^{m}} f(y) d y
$$

Definition 4. Let $B_{t}^{\delta}\left(\hat{f)(\xi)}=\left(1-t^{2}|\xi|^{2}\right)_{+}^{\delta} \hat{f}(\xi)\right.$. We denote

$$
B_{\delta, t}^{A}(f)(x)=\int_{R^{n}} \frac{R_{m+1}(A ; x, y)}{|x-y|^{m}} B_{t}^{\delta}(x-y) f(y) d y,
$$

where $B_{t}^{\delta}(z)=t^{-n} B^{\delta}(z / t)$ for $t>0$. The maximal multilinear Bochner-Riesz operator is defined by

$$
B_{\delta, *}^{A}(f)(x)=\sup _{t>0}\left|B_{\delta, t}^{A}(f)(x)\right|
$$

We also define

$$
B_{*}^{\delta}(f)(x)=\sup _{t>0}\left|B_{t}^{\delta}(f)(x)\right|,
$$

which is the maximal Bochner-Riesz operator (see [12][14][15]). The variant of $B_{\delta, *}^{A}$ is defined by

$$
\tilde{B}_{\delta, *}^{A}(f)(x)=\sup _{t>0}\left|\tilde{B}_{\delta, t}^{A}(f)(x)\right|
$$

where

$$
\tilde{B}_{\delta, t}^{A}(f)(x)=\int_{R^{n}} \frac{Q_{m+1}(A ; x, y)}{|x-y|^{m}} B_{t}^{\delta}(x-y) f(y) d y .
$$

For $g_{\psi}$, let $H$ be the space $H=\left\{h:\|h\|=\left(\int_{0}^{\infty}|h(t)|^{2} d t / t\right)^{1 / 2}<\infty\right\}$. Then for each fixed $x \in R^{n}, F_{t}^{A}(f)(x)$ and $F_{t}(f)(x)$ may be viewed as the mapping from $[0,+\infty)$ to $H$. It is clear that

$$
g_{\psi}(f)(x)=\left\|F_{t}(f)(x)\right\| \text { and } g_{\psi}^{A}(f)(x)=\left\|F_{t}^{A}(f)(x)\right\| ;
$$

For $\mu_{\Omega}$, let $H$ be the space $H=\left\{h:|| h||=\left(\int_{0}^{\infty}|h(t)|^{2} d t / t^{3}\right)^{1 / 2}<\infty\right\}$. Then for each fixed $x \in R^{n}, F_{t}^{A}(f)(x)$ and $F_{t}(f)(x)$ may be viewed as the mapping from $(0,+\infty)$ to $H$, and it is clear that

$$
\mu_{\Omega}^{A}(f)(x)=\left\|F_{t}^{A}(f)(x)\right\| \text { and } \mu_{\Omega}(f)(x)=\left\|F_{t}(f)(x)\right\| ;
$$


For $B_{\delta, *}$, let $H$ be the space $H=\left\{h:\|h\|=\sup _{t>0}|h(t)|<\infty\right\}$. Then it is clear that

$$
B_{*}^{\delta}(f)(x)=\left\|B_{t}^{\delta}(f)(x)\right\| \text { and } B_{\delta, *}^{A}(f)(x)=\left\|B_{\delta, t}^{A}(f)(x)\right\| .
$$

It is obvious that Definition 2, 3 and 4 are the particular examples of Definition 1. Note that when $m=0, T^{A}, g_{\psi}^{A}, \mu_{\Omega}^{A}$ and $B_{\delta, *}^{A}$ is just the commutators generated by $F_{t}$ and $A$ (see[12-14][18]). It is well-known that multilinear operator, as an extension of commutator, is of great interest in harmonic analysis and has been widely studied by many authors (see [3-7, 9]).

We shall prove the following theorems in Section 3.

Theorem 1. Let $w \in A_{1}$ and $D^{\alpha} A \in B M O\left(R^{n}\right)$ for all $\alpha$ with $|\alpha|=m$. Then

(i) $g_{\psi}^{A}$ is bounded from $L^{\infty}(w)$ to $B M O(w)$;

(i) $\tilde{g}_{\psi}^{A}$ is bounded from $H^{1}(w)$ to $L^{1}(w)$;

(iii) $g_{\psi}^{A}$ is bounded from $H^{1}(w)$ to weak $L^{1}(w)$;

(iv) If for any $H^{1}(w)$-atom a supported on certain cube $Q$ and $u \in 3 Q \backslash 2 Q$, there is

$$
\int_{(4 Q)^{c}}\left\|\sum_{|\alpha|=m} \frac{1}{\alpha !} \frac{(x-u)^{\alpha}}{|x-u|^{m}} \psi_{t}(x-u) \int_{Q} D^{\alpha} A(y) a(y) d y\right\| w(x) d x \leq C,
$$

then $g_{\psi}^{A}$ is bounded from $H^{1}(w)$ to $L^{1}(w)$;

(v) If for any cube $Q$ and $u \in 3 Q \backslash 2 Q$, there is

$$
\begin{aligned}
& \frac{1}{w(Q)} \int_{Q}\left\|\sum_{|\alpha|=m} \frac{1}{\alpha !}\left(D^{\alpha} A(x)-\left(D^{\alpha} A\right)_{Q}\right) \int_{(4 Q)^{c}} \frac{(u-y)^{\alpha}}{|u-y|^{m}} \psi_{t}(u-y) f(y) d y\right\| \\
& w(x) d x \leq C\|f\|_{L^{\infty}(w)},
\end{aligned}
$$

then $\tilde{g}_{\psi}^{A}$ is bounded from $L^{\infty}(w)$ to $B M O(w)$.

Theorem 2. Let $w \in A_{1}$ and $D^{\alpha} A \in B M O\left(R^{n}\right)$ for all $\alpha$ with $|\alpha|=m$. Then

(i) $\mu_{\Omega}^{A}$ is bounded from $L^{\infty}(w)$ to $B M O(w)$;

(ii) $\tilde{\mu}_{\Omega}^{A}$ is bounded from $H^{1}(w)$ to $L^{1}(w)$;

(ii) $\mu_{\Omega}^{A}$ is bounded from $H^{1}(w)$ to weak $L^{1}(w)$. 
(iv) If for any $H^{1}(w)$-atom a supported on certain cube $Q$ and $u \in 3 Q \backslash 2 Q$, there is

$$
\int_{(4 Q)^{c}}\left\|\sum_{|\alpha|=m} \frac{1}{\alpha !} \frac{(x-u)^{\alpha}}{|x-u|^{m}} \frac{\Omega(x-u)}{|x-u|^{n-1}} \chi_{\Gamma(x)}(u, t) \int_{Q} D^{\alpha} A(y) a(y) d y\right\| w(x) d x \leq C,
$$

then $\mu_{\Omega}^{A}$ is bounded from $H^{1}(w)$ to $L^{1}(w)$;

(v) If for any cube $Q$ and $u \in 3 Q \backslash 2 Q$, there is

$$
\begin{aligned}
& \frac{1}{w(Q)} \int_{Q} \| \sum_{|\alpha|=m} \frac{1}{\alpha !}\left(D^{\alpha} A(x)-\left(D^{\alpha} A\right)_{Q}\right) \\
& \quad \int_{(4 Q)^{c}} \frac{(u-y)^{\alpha}}{|u-y|^{m}} \frac{\Omega(u-y) \chi_{\Gamma(u)}(y, t)}{|u-y|^{n-1}} f(y) d y\|w(x) d x \leq C\| f \|_{L^{\infty}(w)},
\end{aligned}
$$

then $\tilde{\mu}_{\Omega}^{A}$ is bounded from $L^{\infty}(w)$ to $B M O(w)$.

Theorem 3. Let $w \in A_{1}$ and $D^{\alpha} A \in B M O\left(R^{n}\right)$ for all $\alpha$ with $|\alpha|=m$. If $\delta>(n-1) / 2$, then

(i) $B_{\delta, *}^{A}$ is bounded from $L^{\infty}(w)$ to $B M O(w)$;

(ii) $\tilde{B}_{\delta, *}^{A}$ is bounded from $H^{1}(w)$ to $L^{1}(w)$;

(iii) $B_{\delta, *}^{A}$ is bounded from $H^{1}(w)$ to weak $L^{1}(w)$.

(iv) If for any $H^{1}(w)$-atom a supported on certain cube $Q$ and $u \in 3 Q \backslash 2 Q$, there is

$$
\int_{(4 Q)^{c}}\left\|\sum_{|\alpha|=m} \frac{1}{\alpha !} \frac{(x-u)^{\alpha}}{|x-u|^{m}} B_{t}^{\delta}(x-u) \int_{Q} D^{\alpha} A(y) a(y) d y\right\| w(x) d x \leq C,
$$

then $B_{\delta, *}^{A}$ is bounded from $H^{1}(w)$ to $L^{1}(w)$;

(v) If for any cube $Q$ and $u \in 3 Q \backslash 2 Q$, there is

$$
\begin{aligned}
& \frac{1}{w(Q)} \int_{Q}\left\|\sum_{|\alpha|=m} \frac{1}{\alpha !}\left(D^{\alpha} A(x)-\left(D^{\alpha} A\right)_{Q}\right) \int_{(4 Q)^{c}} \frac{(u-y)^{\alpha}}{|u-y|^{m}} B_{t}^{\delta}(u-y) f(y) d y\right\| \\
& w(x) d x \leq C|| f \|_{L^{\infty}(w)},
\end{aligned}
$$

then $\tilde{B}_{\delta, *}^{A}$ is bounded from $L^{\infty}(w)$ to $B M O(w)$. 
Remark. In general, $g_{\psi}^{A}, \mu_{\Omega}^{A}$ and $B_{\delta, *}^{A}$ are not $\left(H^{1}, L^{1}\right)$ bounded.

\section{Main Teorem And Proof}

We first prove a general theorem.

Main Theorem. Let $w \in A_{1}$ and $D^{\alpha} A \in B M O\left(R^{n}\right)$ for all $\alpha$ with $|\alpha|=m$. Suppose that $F_{t}, T, T^{A}$ are the same as in Definition 1 and that $T$ is bounded on $L^{p}(w)$ for any $1<p \leq \infty$. If $T$ satisfies the size condition:

$$
\left\|F_{t}^{A}(f)(x)-F_{t}^{A}(f)\left(x_{0}\right)\right\|\|\leq C\| D^{\alpha} A\left\|_{B M O}\right\| f \|_{L^{\infty}(w)}
$$

for any cube $Q$ with supp $\subset(2 Q)^{c}$ and $x \in Q$. Then $T^{A}$ is bounded from $B M O(w)$ to $L^{\infty}(w)$.

To prove the theorem, we need the following lemma.

Lemma 1. (see [6]) Let $A$ be a function on $R^{n}$ and $D^{\alpha} A \in L^{q}\left(R^{n}\right)$ for all $\alpha$ with $|\alpha|=m$ and some $q>n$. Then

$$
\left|R_{m}(A ; x, y)\right| \leq C|x-y|^{m} \sum_{|\alpha|=m}\left(\frac{1}{|\tilde{Q}(x, y)|} \int_{\tilde{Q}(x, y)}\left|D^{\alpha} A(z)\right|^{q} d z\right)^{1 / q}
$$

where $\tilde{Q}(x, y)$ is the cube centered at $x$ and having side length $5 \sqrt{n}|x-y|$.

Proof of Main Theorem. It is only to prove that there exists a constant $C_{Q}$ such that

$$
\frac{1}{w(Q)} \int_{Q}\left|T^{A}(f)(x)-C_{Q}\right| w(x) d x \leq C|| f \|_{L^{\infty}(w)}
$$

holds for any cube $Q$. Fix a cube $Q=Q\left(x_{0}, d\right)$. Let $\tilde{Q}=5 \sqrt{n} Q$ and $\tilde{A}(x)=$ $A(x)-\sum_{|\alpha|=m} \frac{1}{\alpha !}\left(D^{\alpha} A\right)_{\tilde{Q}} x^{\alpha}$, then $R_{m}(A ; x, y)=R_{m}(\tilde{A} ; x, y)$ and $D^{\alpha} \tilde{A}=D^{\alpha} A-$ $\left(D^{\alpha} A\right)_{\tilde{Q}}$ for $|\alpha|=m$. We write, for $f_{1}=f \chi_{\tilde{Q}}$ and $f_{2}=f \chi_{R^{n} \backslash \tilde{Q}}$,

$$
\begin{aligned}
F_{t}^{A}(f)(x)= & \int_{R^{n}} \frac{R_{m}(\tilde{A} ; x, y)}{|x-y|^{m}} F(x, y, t) f_{1}(y) d y \\
& -\sum_{|\alpha|=m} \frac{1}{\alpha !} \int_{R^{n}} \frac{F(x, y, t)(x-y)^{\alpha}}{|x-y|^{m}} D^{\alpha} \tilde{A}(y) f_{1}(y) d y \\
& +\int_{R^{n}} \frac{R_{m+1}(\tilde{A} ; x, y)}{|x-y|^{m}} F(x, y, t) f_{2}(y) d y
\end{aligned}
$$


then

$$
\begin{aligned}
& \left|T^{A}(f)(x)-T^{\tilde{A}}\left(f_{2}\right)\left(x_{0}\right)\right|=\left|\left\|F_{t}^{A}(f)(x)\right\|-\left\|F_{t}^{\tilde{A}}(f)\left(x_{0}\right)\right\|\right| \\
\leq & \left\|F_{t}^{A}(f)(x)-F_{t}^{\tilde{A}}(f)\left(x_{0}\right)\right\| \\
\leq & \left\|F_{t}\left(\frac{R_{m}(\tilde{A} ; x, \cdot)}{|x-\cdot|^{m}} f_{1}\right)(x)\right\| \\
& +\sum_{|\alpha|=m} \frac{1}{\alpha !}\left\|F_{t}\left(\frac{(x-\cdot)^{\alpha}}{|x-\cdot|^{m}} D^{\alpha} \tilde{A} f_{1}\right)(x)\right\|+\left\|F_{t}^{\tilde{A}}\left(f_{2}\right)(x)-F_{t}^{\tilde{A}}\left(f_{2}\right)\left(x_{0}\right)\right\| \\
:= & I(x)+I I(x)+\operatorname{III}(x),
\end{aligned}
$$

thus,

$$
\begin{aligned}
& \frac{1}{w(Q)} \int_{Q}\left|T^{A}(f)(x)-T^{\tilde{A}}(f)\left(x_{0}\right)\right| w(x) d x \\
\leq & \frac{1}{w(Q)} \int_{Q} I(x) w(x) d x+\frac{1}{w(Q)} \int_{Q} I I(x) w(x) d x+\frac{1}{w(Q)} \int_{Q} I I I(x) w(x) d x \\
:= & I+I I+I I I .
\end{aligned}
$$

Now, let us estimate $I, I I$ and $I I I$, respectively. First, for $x \in Q$ and $y \in \tilde{Q}$, using Lemma 1, we get

$$
R_{m}(\tilde{A} ; x, y) \leq C|x-y|^{m} \sum_{|\alpha|=m}\left\|D^{\alpha} A\right\|_{B M O}
$$

thus, by the $L^{\infty}(w)$-boundedness of $T$, we get

$$
\begin{aligned}
I & \leq \frac{C}{w(Q)} \int_{Q}\left|T\left(\sum_{|\alpha|=m}\left\|D^{\alpha} A\right\|_{B M O} f_{1}\right)(x)\right| w(x) d x \\
& \leq C \sum_{|\alpha|=m}\left\|D^{\alpha} A\right\|_{B M O}\left\|T\left(f_{1}\right)\right\|_{L^{\infty}(w)} \\
& \leq C \sum_{|\alpha|=m}\left\|D^{\alpha} A\right\|_{B M O}\|f\|_{L^{\infty}(w)} ;
\end{aligned}
$$

Secondly, since $w \in A_{1}, w$ satisfies the reverse of Hölder' inequality:

$$
\left(\frac{1}{|Q|} \int_{Q} w(x)^{q} d x\right)^{1 / q} \leq \frac{C}{|Q|} \int_{Q} w(x) d x
$$

for all cube $Q$ and some $1<q<\infty$ (see[10]), thus, taking $p>1$, by the $L^{p}(w)$ boundedness of $T$ and the Hölder' inequality, we gain 


$$
\begin{aligned}
I I \leq & \frac{C}{w(Q)} \int_{Q}\left|T\left(\sum_{|\alpha|=m}\left(D^{\alpha} A-\left(D^{\alpha} A\right)_{\tilde{Q}}\right) f_{1}\right)(x)\right| w(x) d x \\
\leq & C \sum_{|\alpha|=m}\left(\frac{1}{w(Q)} \int_{Q}\left|T\left(\left(D^{\alpha} A-\left(D^{\alpha} A\right)_{\tilde{Q}}\right) f_{1}\right)(x)\right|^{p} w(x) d x\right)^{1 / p} \\
\leq & C \sum_{|\alpha|=m}\left(\frac{1}{w(Q)} \int\left|\left(D^{\alpha} A(x)-\left(D^{\alpha} A\right)_{\tilde{Q}}\right) f_{1}(x)\right|^{p} w(x) d x\right)^{1 / p} \\
\leq & C \sum_{|\alpha|=m} w(Q)^{-1 / p}\left(\int_{\tilde{Q}}\left|D^{\alpha} A(x)-\left(D^{\alpha} A\right)_{\tilde{Q}}\right|^{p q^{\prime}} d x\right)^{1 / p q^{\prime}} \\
& \left(\int_{\tilde{Q}} w(x)^{q} d x\right)^{1 / p q}\|f\|_{L^{\infty}(w)} \\
\leq & C \sum_{|\alpha|=m}\left(\frac{1}{|Q|} \int_{\tilde{Q}}\left|D^{\alpha} A(x)-\left(D^{\alpha} A\right)_{\tilde{Q}}\right|^{p q^{\prime}} d x\right)^{1 / p q^{\prime}}\left(\frac{1}{|Q|} \int_{\tilde{Q}} w(x)^{q} d x\right)^{1 / p q} \\
& \left(\frac{|Q|}{w(Q)}\right)^{1 / p}\|f\|_{L^{\infty}(w)} \\
\leq & C \sum_{|\alpha|=m}\left\|D^{\alpha} A\right\|_{B M O}\left(\frac{1}{|Q|} \int_{\tilde{Q}} w(x) d x\right)^{1 / p}\left(\frac{|Q|}{w(Q)}\right)^{1 / p}\|f\|_{L^{\infty}(w)} \\
\leq & C \sum_{|\alpha|=m}\left\|D^{\alpha} A\right\|_{B M O}\|f\|_{L^{\infty}(w)}
\end{aligned}
$$

For $I I I$, by the size condition of $T$, we have

$$
I I I \leq C \sum_{|\alpha|=m}\left\|D^{\alpha} A\right\|_{B M O}\|f\|_{L^{\infty}(w)} .
$$

This completes the proof of Main theorem.

To prove Theorem 1, 2 and 3, we need the following lemma.

Lemma 2. Let $w \in A_{1}, 1<p<\infty, \delta>(n-1) / 2$ and $D^{\alpha} A \in B M O\left(R^{n}\right)$ for all $\alpha$ with $|\alpha|=m$. Then $g_{\psi}^{A}, \mu_{\Omega}^{A}$ and $B_{*, \delta}^{A}$ are all bounded on $L^{p}(w)$.

Proof. By Minkowski' inequality, we get

$$
\begin{aligned}
g_{\psi}^{A}(f)(x) & \leq \int_{R^{n}} \frac{|f(y)|\left|R_{m+1}(A ; x, y)\right|}{|x-y|^{m}}\left(\int_{0}^{\infty}\left|\psi_{t}(x-y)\right|^{2} \frac{d t}{t}\right)^{1 / 2} d y \\
& \leq C \int_{R^{n}} \frac{|f(y)|\left|R_{m+1}(A ; x, y)\right|}{|x-y|^{m}}\left(\int_{0}^{\infty} \frac{t^{-2 n}}{(1+|x-y| / t)^{2(n+1)}} \frac{d t}{t}\right)^{1 / 2} d y
\end{aligned}
$$




$$
\begin{aligned}
& \leq C \int_{R^{n}} \frac{\left|R_{m+1}(A ; x, y)\right|}{|x-y|^{m+n}}|f(y)| d y \\
\mu_{\Omega}^{A}(f)(x) & \leq \int_{R^{n}} \frac{|\Omega(x-y)|\left|R_{m+1}(A ; x, y)\right|}{|x-y|^{m+n-1}}|f(y)|\left(\int_{|x-y|}^{\infty} \frac{d t}{t^{3}}\right)^{1 / 2} d y \\
& \leq C \int_{R^{n}} \frac{\left|R_{m+1}(A ; x, y)\right|}{|x-y|^{m+n}}|f(y)| d y,
\end{aligned}
$$

and

$$
\begin{aligned}
& \left|B_{r}^{\delta}(x-y)\right| \leq C r^{-n}(1+|x-y| / r)^{-(\delta+(n+1) / 2)} \\
= & C\left(\frac{r}{r+|x-y|}\right)^{\delta-(n-1) / 2} \frac{1}{(r+|x-y|)^{n}} \leq|x-y|^{-n},
\end{aligned}
$$

thus,

$$
B_{*, \delta}^{A}(f)(x) \leq C \int_{R^{n}} \frac{\left|R_{m+1}(A ; x, y)\right|}{|x-y|^{m+n}}|f(y)| d y,
$$

so that, the lemma follows from [9].

Proof of Theorem 1. (i) First, by the proof of Lemma 2, we get

$$
g_{\psi}(f)(x) \leq C \int_{R^{n}} \frac{|f(y)|}{|x-y|^{n}} d y,
$$

thus, $g_{\psi}$ is $L^{p}(w)$-bounded for $p>1$ by [2]. Now, it suffices to verify that $g_{\psi}$ satisfies the size condition in Main Theorem. For supp $f \subset(2 Q)^{c}$, let $\tilde{A}(x)=$ $A(x)-\sum_{|\alpha|=m} \frac{1}{\alpha !}\left(D^{\alpha} A\right)_{Q} x^{\alpha}$.

Write

$$
\begin{aligned}
& F_{t}^{\tilde{A}}(f)(x)-F_{t}^{\tilde{A}}(f)\left(x_{0}\right)=\int_{R^{n}}\left[\frac{\psi_{t}(x-y)}{|x-y|^{m}}-\frac{\psi_{t}\left(x_{0}-y\right)}{\left|x_{0}-y\right|^{m}}\right] R_{m}(\tilde{A} ; x, y) f(y) d y \\
& \quad+\int_{R^{n}} \frac{\psi_{t}\left(x_{0}-y\right) f(y)}{\left|x_{0}-y\right|^{m}}\left[R_{m}(\tilde{A} ; x, y)-R_{m}\left(\tilde{A} ; x_{0}, y\right)\right] d y \\
& \quad-\sum_{|\alpha|=m} \frac{1}{\alpha !} \int_{R^{n}}\left(\frac{\psi_{t}(x-y)(x-y)^{\alpha}}{|x-y|^{m}}-\frac{\psi_{t}\left(x_{0}-y\right)\left(x_{0}-y\right)^{\alpha}}{\left|x_{0}-y\right|^{m}}\right) D^{\alpha} \tilde{A}(y) f(y) d y \\
& \quad:=I_{1}+I_{2}+I_{3} .
\end{aligned}
$$

Note that $|x-y| \sim\left|x_{0}-y\right|$ for $x \in Q$ and $y \in R^{n} \backslash Q$. By Lemma 1 and the following inequality (see [16])

$$
\left|b_{Q_{1}}-b_{Q_{2}}\right| \leq\left. C \log \left(\left|Q_{2}\right| /\left|Q_{1}\right|\right)|| b\right|_{B M O} \text { for } Q_{1} \subset Q_{2},
$$


we know that, for $x \in Q$ and $y \in 2^{k+1} \tilde{Q} \backslash 2^{k} \tilde{Q}$,

$$
\begin{aligned}
& \left|R_{m}(\tilde{A} ; x, y)\right| \leq C|x-y|^{m} \sum_{|\alpha|=m}\left(|| D^{\alpha} A \|_{B M O}+\left|\left(D^{\alpha} A\right)_{\tilde{Q}(x, y)}-\left(D^{\alpha} A\right)_{\tilde{Q}}\right|\right) \\
\leq & C k|x-y|^{m} \sum_{|\alpha|=m}|| D^{\alpha} A \|_{B M O}
\end{aligned}
$$

Thus, similar to the proof of Lemma 2, we get

$$
\begin{aligned}
\left\|I_{1}\right\| & \leq C \int_{R^{n} \backslash \tilde{Q}}\left(\frac{\left|x-x_{0}\right|}{\left|x_{0}-y\right|^{m+n+1}}+\frac{\left|x-x_{0}\right|^{\varepsilon}}{\left|x_{0}-y\right|^{m+n+\varepsilon}}\right)\left|R_{m}(\tilde{A} ; x, y) \| f(y)\right| d y \\
& \leq C \sum_{|\alpha|=m}\left\|D^{\alpha} A\right\|_{B M O} \sum_{k=0}^{\infty} \int_{2^{k+1} \tilde{Q} \backslash 2^{k} \tilde{Q}} k\left(\frac{\left|x-x_{0}\right|}{\left|x_{0}-y\right|^{n+1}}+\frac{\left|x-x_{0}\right|^{\varepsilon}}{\left|x_{0}-y\right|^{n+\varepsilon}}\right)|f(y)| d y \\
& \leq C \sum_{|\alpha|=m}\left\|D^{\alpha} A\right\|_{B M O}\|f\|_{L^{\infty}(w)} \sum_{k=1}^{\infty} k\left(2^{-k}+2^{-\varepsilon k}\right) \\
& \leq C \sum_{|\alpha|=m}\left\|D^{\alpha} A\right\|_{B M O}\|f\|_{L^{\infty}(w)}
\end{aligned}
$$

For $I_{2}$, by the formula (see [6]):

$$
R_{m}(\tilde{A} ; x, y)-R_{m}\left(\tilde{A} ; x_{0}, y\right)=\sum_{|\beta|<m} \frac{1}{\beta !} R_{m-|\beta|}\left(D^{\beta} \tilde{A} ; x, x_{0}\right)(x-y)^{\beta}
$$

and Lemma 1, we have

$$
\left|R_{m}(\tilde{A} ; x, y)-R_{m}\left(\tilde{A} ; x_{0}, y\right)\right| \leq C \sum_{|\beta|<m} \sum_{|\alpha|=m}\left|x-x_{0}\right|^{m-|\beta|}|x-y|^{|\beta|}|| D^{\alpha} A||_{B M O}
$$

similar to the estimates of $I_{1}$, we get

$$
\begin{aligned}
\left\|I_{2}\right\| & \leq C \sum_{|\alpha|=m}\left\|D^{\alpha} A\right\|_{B M O} \sum_{k=0}^{\infty} \int_{2^{k+1} \tilde{Q} \backslash 2^{k} \tilde{Q}} \frac{\left|x-x_{0}\right|}{\left|x_{0}-y\right|^{n+1}}|f(y)| d y \\
& \leq C \sum_{|\alpha|=m}\left\|D^{\alpha} A\right\|_{B M O}\|f\|_{L^{\infty}(w)}
\end{aligned}
$$

For $I_{3}$, similar to the estimates of $I_{1}$, we get

$$
\begin{aligned}
\left\|I_{3}\right\| & \leq C \sum_{|\alpha|=m} \sum_{k=0}^{\infty} \int_{2^{k+1} \tilde{Q} \backslash 2^{k} \tilde{Q}}\left(\frac{\left|x-x_{0}\right|}{\left|x_{0}-y\right|^{n+1}}+\frac{\left|x-x_{0}\right|^{\varepsilon}}{\left|x_{0}-y\right|^{n+\varepsilon}}\right)\left|D^{\alpha} \tilde{A}(y) \| f(y)\right| d y \\
& \leq C \sum_{|\alpha|=m} \sum_{k=1}^{\infty}\left(2^{-k}+2^{-\varepsilon k}\right)\left(\left|2^{k} \tilde{Q}\right|^{-1} \int_{2^{k} \tilde{Q}}\left|D^{\alpha} A(y)-\left(D^{\alpha} A\right)_{\tilde{Q}}\right| d y\right)\|f\|_{L^{\infty}(w)} \\
& \leq C \sum_{|\alpha|=m}\left\|D^{\alpha} A\right\|_{B M O}\|f\|_{L^{\infty}(w)} .
\end{aligned}
$$


Thus

$$
\left\|F_{t}^{\tilde{A}}\left(f_{2}\right)(x)-F_{t}^{\tilde{A}}\left(f_{2}\right)\left(x_{0}\right)\right\| \leq C \sum_{|\alpha|=m}\left\|D^{\alpha} A\right\|_{B M O}\|f\|_{L^{n / \delta}} .
$$

(ii). It suffices to show that there exists a constant $C>0$ such that for every $H^{1}(w)$-atom $a$, we have

$$
\left\|\tilde{g}_{\psi}^{A}(a)\right\|_{L^{1}(w)} \leq C
$$

We write

$$
\int_{R^{n}} \tilde{g}_{\psi}^{A}(a)(x) w(x) d x=\left[\int_{2 Q}+\int_{(2 Q)^{c}}\right] \tilde{g}_{\psi}^{A}(a)(x) w(x) d x:=J+J J .
$$

For $J$, by the following equality

$$
Q_{m+1}(A ; x, y)=R_{m+1}(A ; x, y)+\sum_{|\alpha|=m} \frac{1}{\alpha !}(x-y)^{\alpha}\left(D^{\alpha} A(x)-D^{\alpha} A(y)\right),
$$

we have, similar to the proof of Lemma 2,

$$
\tilde{g}_{\psi}^{A}(a)(x) \leq g_{\psi}^{A}(a)(x)+C \sum_{|\alpha|=m} \int_{R^{n}} \frac{\left|D^{\alpha} A(x)-D^{\alpha} A(y)\right|}{|x-y|^{n}}|a(y)| d y,
$$

thus, $\tilde{g}_{\psi}^{A}$ is $L^{\infty}(w)$-bounded by Lemma 2 and [2]. We get

$$
J \leq C\left\|\tilde{g}_{\delta}^{A}(a)\right\|_{L^{\infty}(w)} w(2 Q) \leq C\|a\|_{L^{\infty}(w)} w(Q) \leq C .
$$

To obtain the estimate of $J J$, we denote that $\tilde{A}(x)=A(x)-\sum_{|\alpha|=m} \frac{1}{\alpha !}\left(D^{\alpha} A\right)_{2 Q} x^{\alpha}$. Then $Q_{m}(A ; x, y)=Q_{m}(\tilde{A} ; x, y)$. We write, by the vanishing moment of $a$ and for $x \in(2 Q)^{c}$,

$$
\begin{aligned}
\tilde{F}_{t}^{A}(a)(x)= & \int_{R^{n}} \frac{\psi_{t}(x-y) R_{m}(A ; x, y)}{|x-y|^{m}} a(y) d y \\
& -\sum_{|\alpha|=m} \frac{1}{\alpha !} \int_{R^{n}} \frac{\psi_{t}(x-y) D^{\alpha} \tilde{A}(x)(x-y)^{\alpha}}{|x-y|^{m}} a(y) d y \\
= & \int_{R^{n}}\left[\frac{\psi_{t}(x-y)}{|x-y|^{m}}-\frac{\psi_{t}\left(x-x_{0}\right)}{\left|x-x_{0}\right|^{m}}\right] R_{m}(\tilde{A} ; x, y) a(y) d y \\
& +\int_{R^{n}} \frac{\psi_{t}\left(x-x_{0}\right) a(y)}{\left|x_{0}-x\right|^{m}}\left[R_{m}(\tilde{A} ; x, y)-R_{m}\left(\tilde{A} ; x, x_{0}\right)\right] d y \\
& -\sum_{|\alpha|=m} \frac{1}{\alpha !} \int_{R^{n}}\left[\frac{\psi_{t}(x-y)(x-y)^{\alpha}}{|x-y|^{m}}-\frac{\psi_{t}\left(x-x_{0}\right)\left(x-x_{0}\right)^{\alpha}}{\left|x-x_{0}\right|^{m}}\right] \\
& D^{\alpha} \tilde{A}(x) a(y) d y, \\
:= & J J_{1}+J J_{2}+J J_{3} .
\end{aligned}
$$


Similar to the proof of Lemma 2 and (i), we obtain, for $x \in(2 Q)^{c}$,

$$
\begin{aligned}
\left\|J J_{1}\right\| \leq & C \int_{R^{n}}\left[\frac{\left|y-x_{0}\right|}{|x-y|^{n+m+1}}+\frac{\left|y-x_{0}\right|^{\varepsilon}}{|x-y|^{n+m+\varepsilon}}\right]\left|R_{m}(\tilde{A} ; x, y)\right||a(y)| d y \\
\leq & \left.C \sum_{|\alpha|=m}|| D^{\alpha} A\right|_{B M O} w(Q)^{-1} \\
& \left(|Q|^{1+1 / n}\left|x-x_{0}\right|^{-n-1}+|Q|^{1+\varepsilon / n}\left|x-x_{0}\right|^{-n-\varepsilon}\right), \\
\left\|J J_{2}\right\| \leq & C \int_{Q} \frac{\left|R_{m}(\tilde{A} ; x, y)-R_{m}\left(\tilde{A} ; x, x_{0}\right)\right||a(y)|}{|x-y|^{m+n}} d y \\
\leq & \left.C \sum_{|\alpha|=m}|| D^{\alpha} A\right|_{B M O} \int_{Q} \frac{\left|x_{0}-y\right||a(y)|}{\left|x-x_{0}\right|^{n+1}} d y \\
\leq & \left.C \sum_{|\alpha|=m}|| D^{\alpha} A\right|_{B M O} w(Q)^{-1}|Q|^{1+1 / n}\left|x-x_{0}\right|^{-n-1}, \\
|| J J_{3} \| \leq & C \int_{Q} \frac{\left|x_{0}-y\right|}{|x-y|^{n+1}} \sum_{|\alpha|=m}\left|D^{\alpha} \tilde{A}(x)\right||a(y)| d y \\
\leq & C \sum_{|\alpha|=m}\left|D^{\alpha} \tilde{A}(x)\right| w(Q)^{-1} \mid\left(|Q|^{1+1 / n}\left|x-x_{0}\right|^{-n-1}+|Q|^{1+\varepsilon / n}\left|x-x_{0}\right|^{-n-\varepsilon}\right) .
\end{aligned}
$$

Note that if $w \in A_{1}$, then $\frac{w\left(Q_{2}\right)}{\left|Q_{2}\right|} \frac{\left|Q_{1}\right|}{w\left(Q_{1}\right)} \leq C$ for all cubes $Q_{1}, Q_{2}$ with $Q_{1} \subset Q_{2}$. Thus, by the Hölder' inequality and the reverse of Hölder' inequality for $w \in A_{1}$, we obtain

$$
\begin{aligned}
J J & \leq \int_{(2 Q)^{c}}\left\|J J_{1}+J J_{2}+J J_{3}\right\| w(x) d x \\
& \leq C \sum_{|\alpha|=m}\left\|D^{\alpha} A\right\|_{B M O} \sum_{k=1}^{\infty}\left(2^{-k}+2^{-\varepsilon k}\right)\left(\frac{|Q|}{w(Q)} \frac{w\left(2^{k+1} Q\right)}{\left|2^{k+1} Q\right|}\right) \\
& +C \sum_{|\alpha|=m} \sum_{k=1}^{\infty}\left(2^{-k}+2^{-\varepsilon k}\right) \frac{|Q|}{w(Q)}\left(\frac{1}{\left|2^{k+1} Q\right|} \int_{2^{k+1} Q}\left|D^{\alpha} \tilde{A}(x)\right|^{q^{\prime}} d x\right)^{1 / q^{\prime}} \\
& \left(\frac{1}{\left|2^{k+1} Q\right|} \int_{2^{k+1} Q} w(x)^{q} d x\right)^{1 / q} \\
& \leq C \sum_{|\alpha|=m}|| D^{\alpha} A \|_{B M O} \sum_{k=1}^{\infty} k\left(2^{-k}+2^{-\varepsilon k}\right)\left(\frac{w\left(2^{k+1} Q\right)}{\left|2^{k+1} Q\right|} \frac{|Q|}{w(Q)}\right) \\
& \leq C .
\end{aligned}
$$

(iii). By the equality 


$$
R_{m+1}(A ; x, y)=Q_{m+1}(A ; x, y)+\sum_{|\alpha|=m} \frac{1}{\alpha !}(x-y)^{\alpha}\left(D^{\alpha} A(x)-D^{\alpha} A(y)\right),
$$

similar to the proof of Lemma 2, we get

$$
g_{\psi}^{A}(f)(x) \leq \tilde{g}_{\psi}^{A}(f)(x)+C \sum_{|\alpha|=m} \int_{R^{n}} \frac{\left|D^{\alpha} A(x)-D^{\alpha} A(y)\right|}{|x-y|^{n}}|f(y)| d y,
$$

by (i)(ii) and [2], we obtain

$$
\begin{aligned}
& w\left(\left\{x \in R^{n}: g_{\psi}^{A}(f)(x)>\lambda\right\}\right) \\
\leq & w\left(\left\{x \in R^{n}: \tilde{g}_{\psi}^{A}(f)(x)>\lambda / 2\right\}\right) \\
& +w\left(\left\{x \in R^{n}: \sum_{|\alpha|=m} \int_{R^{n}} \frac{\left|D^{\alpha} A(x)-D^{\alpha} A(y)\right|}{|x-y|^{n}}|f(y)| d y>C \lambda\right\}\right) \\
\leq & C\|f\|_{H^{1}(w)} / \lambda .
\end{aligned}
$$

(iv). Let $a$ be $H^{1}(w)$-atom with supp $a \subset Q=Q\left(x_{0}, d\right)$. We write, by the vanishing moment of $a$ and for $u \in 3 Q \backslash 2 Q$,

$$
\begin{aligned}
& F_{t}^{A}(a)(x)=\chi_{4 Q}(x) F_{t}^{A}(a)(x)+\chi_{(4 Q)^{c}}(x) \\
& \int_{R^{n}}\left[\frac{R_{m}(\tilde{A} ; x, y) \psi_{t}(x-y)}{|x-y|^{m}}-\frac{R_{m}(\tilde{A} ; x, u) \psi_{t}(x-u)}{|x-u|^{m}}\right] a(y) d y \\
& -\chi_{(4 Q)^{c}}(x) \sum_{|\alpha|=m} \frac{1}{\alpha !} \\
& \int_{R^{n}}\left[\frac{\psi_{t}(x-y)(x-y)^{\alpha}}{|x-y|^{m}}-\frac{\psi_{t}(x-u)(x-u)^{\alpha}}{|x-u|^{m}}\right] D^{\alpha} \tilde{A}(y) a(y) d y \\
& -\chi_{(4 Q)^{c}}(x) \sum_{|\alpha|=m} \frac{1}{\alpha !} \int_{R^{n}} \frac{(x-u)^{\alpha}}{|x-u|^{m}} \psi_{t}(x-u) D^{\alpha} \tilde{A}(y) a(y) d y,
\end{aligned}
$$

then

$$
\begin{aligned}
& g_{\psi}^{A}(a)(x)=\left\|F_{t}^{A}(a)(x)\right\| \leq \chi_{4 Q}(x)\left\|F_{t}^{A}(a)(x)\right\| \\
& +\chi_{(4 Q)^{c}}(x)\left\|\int_{R^{n}}\left[\frac{R_{m}(\tilde{A} ; x, y) \psi_{t}(x-y)}{|x-y|^{m}}-\frac{R_{m}(\tilde{A} ; x, u) \psi_{t}(x-u)}{|x-u|^{m}}\right] a(y) d y\right\| \\
& +\chi_{(4 Q)^{c}}(x)\left\|\sum_{|\alpha|=m} \frac{1}{\alpha !} \int_{R^{n}}\left[\frac{\psi_{t}(x-y)(x-y)^{\alpha}}{|x-y|^{m}}-\frac{\psi_{t}(x-u)(x-u)^{\alpha}}{|x-u|^{m}}\right] D^{\alpha} \tilde{A}(y) a(y) d y\right\|
\end{aligned}
$$




$$
\begin{aligned}
& +\chi_{(4 Q)^{c}}(x)\left\|\sum_{|\alpha|=m} \frac{1}{\alpha !} \int_{R^{n}} \frac{(x-u)^{\alpha}}{|x-u|^{m}} \psi_{t}(x-u) D^{\alpha} \tilde{A}(y) a(y) d y\right\| \\
= & I_{1}(x)+I_{2}(x, u)+I_{3}(x, u)+I_{4}(x, u) .
\end{aligned}
$$

Similar to the proof of (i), we get

$$
\begin{aligned}
& \int_{R^{n}} I_{1}(x) w(x) d x \leq\left\|g_{\psi}^{A}(a)\right\|_{L^{p}(w)} w(4 Q)^{1-1 / p} \leq C\|a\|_{L^{p}(w)} w(Q)^{1-1 / p} \leq C ; \\
& \int_{R^{n}} I_{2}(x, u) w(x) d x \leq C \sum_{k=2}^{\infty} \int_{2^{k+1} Q \backslash 2^{k} Q} \int_{Q}\left(\frac{|y-u|}{|x-y|^{m+n+1}}+\frac{|y-u|^{\varepsilon}}{|x-y|^{m+n+\varepsilon}}\right) \\
& \left|R_{m}(\tilde{A} ; x, y) \| a(y)\right| d y w(x) d x \\
& +\sum_{|\alpha|=m}\left\|D^{\alpha} A\right\|_{B M O} \sum_{k=2}^{\infty} \int_{2^{k+1} Q \backslash 2^{k} Q} \int_{Q} \frac{|y-u|}{|x-y|^{n+1}}|a(y)| \\
& d y w(x) d x \\
& \leq C \sum_{|\alpha|=m}\left\|D^{\alpha} A\right\|_{B M O} \sum_{k=2}^{\infty} \int_{2^{k+1} Q \backslash 2^{k} Q} k \\
& \left(\frac{d}{\left(2^{k} d\right)^{n+1}}+\frac{d^{\varepsilon}}{\left(2^{k} d\right)^{n+\varepsilon}}\right)\|a\|_{L^{\infty}(w)}|Q| w(x) d x \\
& \leq C \sum_{|\alpha|=m}\left\|D^{\alpha} A\right\|_{B M O} \sum_{k=2}^{\infty} k\left(2^{-k}+2^{-\varepsilon k}\right) \frac{w\left(2^{k+1} Q\right)}{\left|2^{k+1} Q\right|} \frac{|Q|}{w(Q)} \\
& \leq C \\
& \int_{(4 Q)^{c}} I_{3}(x, u) w(x) d x \leq C \sum_{|\alpha|=m} \sum_{k=2}^{\infty} \int_{2^{k+1} Q \backslash 2^{k} Q} \int_{Q}\left(\frac{|y-u|}{|x-y|^{n+1}}+\frac{|y-u|^{\varepsilon}}{|x-y|^{n+\varepsilon}}\right) \\
& \left|D^{\alpha} \tilde{A}(y)\right||a(y)| d y w(x) d x \\
& \left.\leq C \sum_{|\alpha|=m k=2}^{\infty} \sum_{\left(2^{k} d\right)^{n+1}}^{\infty}+\frac{d^{\varepsilon}}{\left(2^{k} d\right)^{n+\varepsilon}}\right)\left(\frac{1}{|Q|} \int_{Q}\left|D^{\alpha} \tilde{A}(y)\right| d y\right) \\
& \|\left. a\right|_{L^{\infty}(w)}|Q| w\left(2^{k+1} Q\right) \\
& \leq C \sum_{|\alpha|=m}\left\|D^{\alpha} A\right\|_{B M O} \sum_{k=2}^{\infty}\left(2^{-k}+2^{-\varepsilon k}\right) \frac{w\left(2^{k+1} Q\right)}{\left|2^{k+1} Q\right|} \frac{|Q|}{w(Q)} \\
& \leq C \text {. }
\end{aligned}
$$

Thus, using the condition of $I_{4}(x, u)$, we obtain

$$
\int_{R^{n}} g_{\psi}^{A}(a)(x) w(x) d x \leq C .
$$


(v). For any cube $Q=Q\left(x_{0}, d\right)$, we write, for $f=f \chi_{4 Q}+f \chi_{(4 Q)^{c}}=f_{1}+f_{2}$ and $u \in 3 Q \backslash 2 Q$,

$$
\begin{aligned}
\tilde{F}_{t}^{A}(f)(x)= & \tilde{F}_{t}^{A}\left(f_{1}\right)(x)+\int_{R^{n}} \frac{R_{m}(\tilde{A} ; x, y)}{|x-y|^{m}} \psi_{t}(x-y) f_{2}(y) d y \\
& -\sum_{|\alpha|=m} \frac{1}{\alpha !}\left(D^{\alpha} A(x)-\left(D^{\alpha} A\right)_{Q}\right) \int_{R^{n}} \\
& {\left[\frac{\psi_{t}(x-y)(x-y)^{\alpha}}{|x-y|^{m}}-\frac{\psi_{t}(u-y)(u-y)^{\alpha}}{|u-y|^{m}}\right] f_{2}(y) d y } \\
& -\sum_{|\alpha|=m} \frac{1}{\alpha !}\left(D^{\alpha} A(x)-\left(D^{\alpha} A\right)_{Q}\right) \int_{R^{n}} \frac{(u-y)^{\alpha}}{|u-y|^{m}} \psi_{t}(u-y) f_{2}(y) d y
\end{aligned}
$$

then

$$
\begin{aligned}
& \left|\tilde{g}_{\psi}^{A}(f)(x)-g_{\psi}\left(\frac{R_{m}\left(\tilde{A} ; x_{0}, \cdot\right)}{\left|x_{0}-\cdot\right|^{m}} f_{2}\right)\left(x_{0}\right)\right| \\
= & \left\|\tilde{F}_{t}^{A}(f)(x)\right\|-\left\|F_{t}\left(\frac{R_{m}\left(\tilde{A} ; x_{0}, \cdot\right)}{\left|x_{0}-\cdot\right|^{m}} f_{2}\right)\left(x_{0}\right) \mid\right\| \\
\leq & \left\|\tilde{F}_{t}^{A}(f)(x)-F_{t}\left(\frac{R_{m}\left(\tilde{A} ; x_{0}, \cdot\right)}{\left|x_{0}-\right|^{m}} f_{2}\right)\left(x_{0}\right)\right\| \\
\leq & \left\|\tilde{F}_{t}^{A}\left(f_{1}\right)(x)\right\| \\
& +\left\|\int_{R^{n}}\left[\frac{R_{m}(\tilde{A} ; x, y)}{|x-y|^{m}} \psi_{t}(x-y)-\frac{R_{m}\left(\tilde{A} ; x_{0}, y\right)}{\left|x_{0}-y\right|^{m}} \psi_{t}\left(x_{0}-y\right)\right] f_{2}(y) d y\right\| \\
& +\| \sum_{|\alpha|=m} \frac{1}{\alpha !}\left(D^{\alpha} A(x)-\left(D^{\alpha} A\right)_{Q}\right) \int_{R^{n}} \\
& \left.+\frac{\psi_{t}(x-y)(x-y)^{\alpha}}{|x-y|^{m}}-\frac{\psi_{t}(u-y)(u-y)^{\alpha}}{|u-y|^{m}}\right] f_{2}(y) d y \| \\
& +\left\|\sum_{|\alpha|=m} \frac{1}{\alpha !}\left(D^{\alpha} A(x)-\left(D^{\alpha} A\right)_{Q}\right) \int_{R^{n}} \frac{(u-y)^{\alpha}}{|u-y|^{m}} \psi_{t}(u-y) f_{2}(y) d y\right\| \\
= & J_{1}(x)+J_{2}(x)+J_{3}(x, u)+J_{4}(x, u) .
\end{aligned}
$$

Similar to the proof of (i) and (iv), we get

$$
\frac{1}{w(Q)} \int_{Q} J_{1}(x) w(x) d x \leq w(Q)^{-1 / p}\left\|\tilde{g}_{\psi}^{A}\left(f_{1}\right)\right\|_{L^{p}(w)}
$$




$$
\begin{aligned}
\leq & C w(Q)^{-1 / p}|| f_{1}\left\|_{L^{p}(w)} \leq C|| f\right\|_{L^{\infty}(w)} \\
\frac{1}{w(Q)} \int_{Q} J_{2}(x) w(x) d x \leq & C \frac{1}{w(Q)} \int_{Q} \sum_{k=2}^{\infty} \int_{2^{k+1} Q \backslash 2^{k} Q} k \\
& \left(\frac{\left|x-x_{0}\right|}{\left|x_{0}-y\right|^{n+1}}+\frac{\left|x-x_{0}\right|^{\varepsilon}}{\left|x_{0}-y\right|^{n+\varepsilon}}\right)|f(y)| d y w(x) d x \\
\leq & C|| f\left\|_{L^{\infty}(w)} \sum_{k=1}^{\infty} k\left(2^{-k}+2^{-\varepsilon k}\right) \leq C|| f\right\|_{L^{\infty}(w)} \\
\frac{1}{w(Q)} \int_{Q} J_{3}(x, u) w(x) d x \leq & \left.\left.C \sum_{|\alpha|=m}\left(\frac{1}{|Q|} \int_{Q} \mid D^{\alpha} A(x)-\left(D^{\alpha} A\right)_{Q}\right)\right|^{r^{\prime}} d x\right)^{1 / r^{\prime}} \\
& \left(\frac{1}{|Q|} \int_{Q} w(x)^{r} d x\right)^{1 / r}|Q| w(Q)^{-1} \\
& \times \sum_{k=2}^{\infty}\left(\frac{d}{\left(2^{k} d\right)^{n+1}}+\frac{d^{\varepsilon}}{\left.\left(2^{k} d\right)^{n+\varepsilon}\right)\left|2^{k} Q\right|\|f\|_{L^{\infty}(w)}}\right. \\
\leq & C \sum_{k=2}^{\infty}\left(2^{-k}+2^{-\varepsilon k}\right)\|f\|_{L^{\infty}(w)} \leq C|| f \|_{L^{\infty}(w)} .
\end{aligned}
$$

Thus, using the condition of $J_{4}(x, u)$, we obtain

$$
\frac{1}{w(Q)} \int_{Q}\left|\tilde{g}_{\psi}^{A}(f)(x)-g_{\psi}\left(\frac{R_{m}\left(\tilde{A} ; x_{0}, \cdot\right)}{\left|x_{0}-\cdot\right|^{m}} f_{2}\right)\left(x_{0}\right)\right| w(x) d x \leq C\|f\|_{L^{\infty}(w)} .
$$

This completes the proof of Theorem 1.

Proof of Theorem 2.

(i) First, by the proof of Lemma 2, we get

$$
\mu_{\Omega}(f)(x) \leq C \int_{R^{n}} \frac{|f(y)|}{|x-y|^{n}} d y,
$$

thus, $\mu_{\Omega}$ is $L^{p}(w)$-bounded for $p>1$. Now, it suffices to verify that $\mu_{\Omega}$ satisfies the size condition in Main Theorem. For supp $f \subset(2 Q)^{c}$, let $\tilde{A}(x)$ be the same as the proof of Theorem 1 . We write

$$
\begin{aligned}
& \left\|F_{t}^{\tilde{A}}(f)(x)-F_{t}^{\tilde{A}}(f)\left(x_{0}\right)\right\| \\
\leq & \left(\int_{0}^{\infty} \mid \int_{|x-y| \leq t} \frac{\Omega(x-y) R_{m}(\tilde{A} ; x, y)}{|x-y|^{m+n-1}} f(y) d y\right.
\end{aligned}
$$




$$
\begin{aligned}
& \left.-\left.\int_{\left|x_{0}-y\right| \leq t} \frac{\Omega\left(x_{0}-y\right) R_{m}\left(\tilde{A} ; x_{0}, y\right)}{\left|x_{0}-y\right|^{m+n-1}} f(y) d y\right|^{2} \frac{d t}{t^{3}}\right)^{1 / 2} \\
& +\sum_{|\alpha|=m}\left(\int_{0}^{\infty} \mid \int_{|x-y| \leq t}\left(\frac{\Omega(x-y)(x-y)^{\alpha}}{|x-y|^{m+n-1}}\right.\right. \\
& \left.\left.-\int_{\left|x_{0}-y\right| \leq t} \frac{\Omega\left(x_{0}-y\right)\left(x_{0}-y\right)^{\alpha}}{\left|x_{0}-y\right|^{m+n-1}}\right)\left.D^{\alpha} \tilde{A}(y) f(y) d y\right|^{2} \frac{d t}{t^{3}}\right)^{1 / 2} \\
& \leq\left(\int_{0}^{\infty}\left[\int_{|x-y| \leq t,\left|x_{0}-y\right|>t} \frac{|\Omega(x-y)|\left|R_{m}(\tilde{A} ; x, y)\right|}{|x-y|^{m+n-1}}|f(y)| d y\right]^{2} \frac{d t}{t^{3}}\right)^{1 / 2} \\
& +\left(\int_{0}^{\infty}\left[\int_{|x-y|>t,\left|x_{0}-y\right| \leq t} \frac{\left|\Omega\left(x_{0}-y\right)\right|\left|R_{m}\left(\tilde{A} ; x_{0}, y\right)\right|}{\left|x_{0}-y\right|^{m+n-1}}|f(y)| d y\right]^{2} \frac{d t}{t^{3}}\right)^{1 / 2} \\
& +\left(\int _ { 0 } ^ { \infty } \left[\int_{|x-y| \leq t,\left|x_{0}-y\right| \leq t} \mid \frac{\Omega(x-y) R_{m}(\tilde{A} ; x, y)}{|x-y|^{m+n-1}}\right.\right. \\
& \left.\left.-\frac{\Omega\left(x_{0}-y\right) R_{m}\left(\tilde{A} ; x_{0}, y\right)}{\left|x_{0}-y\right|^{m+n-1}}|| f(y) \mid d y\right]^{2} \frac{d t}{t^{3}}\right)^{1 / 2} \\
& +\sum_{|\alpha|=m}\left(\int_{0}^{\infty} \mid \int_{|x-y| \leq t}\left(\frac{\Omega(x-y)(x-y)^{\alpha}}{|x-y|^{m+n-1}}\right.\right. \\
& \left.\left.-\int_{\left|x_{0}-y\right| \leq t} \frac{\Omega\left(x_{0}-y\right)\left(x_{0}-y\right)^{\alpha}}{\left|x_{0}-y\right|^{m+n-1}}\right)\left.D^{\alpha} \tilde{A}(y) f(y) d y\right|^{2} \frac{d t}{t^{3}}\right)^{1 / 2} \\
& :=K_{1}+K_{2}+K_{3}+K_{4} \text {. }
\end{aligned}
$$

Since $|x-y| \approx\left|x_{0}-y\right|$ when $y \in(2 Q)^{c}$, we get

$$
\begin{aligned}
K_{1} & \leq C \int_{R^{n} \backslash \tilde{Q}} \frac{|f(y)|\left|R_{m}(\tilde{A} ; x, y)\right|}{|x-y|^{m+n-1}}\left(\int_{|x-y| \leq t<\left|x_{0}-y\right|} \frac{d t}{t^{3}}\right)^{1 / 2} d y \\
& \leq C \int_{R^{n} \backslash \tilde{Q}} \frac{|f(y)|\left|R_{m}(\tilde{A} ; x, y)\right|}{|x-y|^{m+n-1}} \frac{\left|x_{0}-x\right|^{1 / 2}}{|x-y|^{3 / 2}} d y \leq C \sum_{|\alpha|=m}\left\|D^{\alpha} A\right\|\left\|_{B M O}\right\| f \|_{L^{\infty}(w)} ;
\end{aligned}
$$

Similarly, we get $K_{2} \leq C \sum_{|\alpha|=m}\left\|D^{\alpha} A\right\|_{B M O}\|f\|_{L^{\infty}(w)}$.

For $K_{3}$, by the following inequality (see [18]): 


$$
\left|\frac{\Omega(x-y)}{|x-y|^{n-1}}-\frac{\Omega\left(x_{0}-y\right)}{\left|x_{0}-y\right|^{n-1}}\right| \leq\left(\frac{\left|x-x_{0}\right|}{\left|x_{0}-y\right|^{n}}+\frac{\left|x-x_{0}\right|^{\gamma}}{\left|x_{0}-y\right|^{n-1+\gamma}}\right),
$$

we gain

$$
\begin{aligned}
K_{3} \leq & C \sum_{|\alpha|=m}\left\|D^{\alpha} A\right\|_{B M O} \int_{R^{n} \backslash \tilde{Q}} \\
& \left(\frac{\left|x-x_{0}\right|}{\left|x_{0}-y\right|^{n}}+\frac{\left|x-x_{0}\right|^{\gamma}}{\left|x_{0}-y\right|^{n-1+\gamma}}\right)\left(\int_{\left|x_{0}-y\right| \leq t,|x-y| \leq t} \frac{d t}{t^{3}}\right)^{1 / 2}|f(y)| d y \\
\leq & C \sum_{|\alpha|=m}\left\|D^{\alpha} A\right\|_{B M O} \sum_{k=1}^{\infty} k\left(2^{-k}+2^{-\gamma k}\right)\|f\|_{L^{\infty}(w)} \\
\leq & C \sum_{|\alpha|=m}\left\|D^{\alpha} A\right\|_{B M O}\|f\|_{L^{\infty}(w)}
\end{aligned}
$$

For $K_{4}$, similar to the proof of $K_{1}, K_{2}$ and $K_{3}$, we obtain

$$
\begin{aligned}
K_{4} \leq & C \sum_{|\alpha|=m} \sum_{k=1}^{\infty} \int_{2^{k+1} \tilde{Q} \backslash 2^{k} \tilde{Q}}\left(\frac{\left|x-x_{0}\right|}{\left|x_{0}-y\right|^{n+1}}+\frac{\left|x-x_{0}\right|^{1 / 2}}{\left|x_{0}-y\right|^{n+1 / 2}}+\frac{\left|x-x_{0}\right|^{\gamma}}{\left|x_{0}-y\right|^{n+\gamma}}\right) \\
& \leq D^{\alpha} \tilde{A}(y)|| f(y) \mid d y \\
\leq & C \sum_{|\alpha|=m}\left\|D^{\alpha} A\right\|_{B M O} \sum_{k=1}^{\infty} k\left(2^{-k}+2^{-k / 2}+2^{-\gamma k}\right)\|f\|_{L^{\infty}(w)} \\
\leq & C \sum_{|\alpha|=m}\left\|D^{\alpha} A\right\|_{B M O}\|f\|_{L^{\infty}(w)} .
\end{aligned}
$$

A same argument as in the proof of Theorem 1 will get the proof of (ii),(iii),(iv) and (v), we omit the details. This completes the proof of Theorem 2.

Proof of Theorem 3.(i) First, by the proof of Lemma 2, we get

$$
B_{*, \delta}(f)(x) \leq C \int_{R^{n}} \frac{|f(y)|}{|x-y|^{n}} d y,
$$

thus, $B_{*, \delta}$ is $L^{p}(w)$-bounded for $p>1$. Now, it suffices to verify that $B_{*, \delta}$ satisfies the size condition in Main Theorem. For supp $f \subset\left(2 Q\left(x_{0}, d\right)\right)^{c}$, let $\tilde{A}(x)$ be the same as the proof of Theorem 1. We write

$$
\begin{aligned}
& B_{t, \delta}^{\tilde{A}}(f)(x)-B_{t, \delta}^{\tilde{A}}(f)\left(x_{0}\right)=\int_{R^{n}}\left[\frac{B_{t}^{\delta}(x-y)}{|x-y|^{m}}-\frac{B_{t}^{\delta}\left(x_{0}-y\right)}{\left|x_{0}-y\right|^{m}}\right] R_{m}(\tilde{A} ; x, y) f(y) d y \\
& \quad+\int_{R^{n}} \frac{B_{t}^{\delta}\left(x_{0}-y\right)}{\left|x_{0}-y\right|^{m}}\left[R_{m}(\tilde{A} ; x, y)-R_{m}\left(\tilde{A} ; x_{0}, y\right)\right] f(y) d y
\end{aligned}
$$




$$
\begin{aligned}
& -\sum_{|\alpha|=m} \frac{1}{\alpha !} \int_{R^{n}}\left(\frac{B_{t}^{\delta}(x-y)(x-y)^{\alpha}}{|x-y|^{m}}-\frac{B_{t}^{\delta}\left(x_{0}-y\right)\left(x_{0}-y\right)^{\alpha}}{\left|x_{0}-y\right|^{m}}\right) D^{\alpha} \tilde{A}(y) f(y) d y \\
= & L_{1}+L_{2}+L_{3} .
\end{aligned}
$$

We consider the following two cases:

Case 1. $0<t \leq d$. In this case, notice that (see [14])

$$
\left|B^{\delta}(z)\right| \leq c(1+|z|)^{-(\delta+(n+1) / 2)},
$$

we obtain

$$
\begin{aligned}
& \left|L_{1}\right| \leq C t^{-n} \int_{R^{n} \backslash \tilde{Q}} \frac{|f(y)|\left|R_{m}(\tilde{A} ; x, y)\right|}{\left|x_{0}-y\right|^{m}}(1+|x-y| / t)^{-(\delta+(n+1) / 2)} d y \\
& \leq C \sum_{|\alpha|=m}\left\|D^{\alpha} A\right\|_{B M O} t^{-n} \sum_{k=0}^{\infty} k \int_{2^{k+1} \tilde{Q} \backslash 2^{k} \tilde{Q}} \mid f(y) \|(1+|x-y| / t)^{-(\delta+(n+1) / 2)} d y \\
& \leq C \sum_{|\alpha|=m}\left\|D^{\alpha} A\right\|_{B M O}(t / d)^{\delta-(n-1) / 2} \sum_{k=1}^{\infty} k 2^{k((n-1) / 2-\delta)}\|f\|_{L^{\infty}(w)} \\
& \leq C \sum_{|\alpha|=m}\left\|D^{\alpha} A\right\|_{B M O}\|f\|_{L^{\infty}(w)}, \\
& \left|L_{2}\right| \leq C t^{-n} \int_{R^{n} \backslash \tilde{Q}} \frac{|f(y)|\left|R_{m}(\tilde{A} ; x, y)-R_{m}\left(\tilde{A} ; x_{0}, y\right)\right|}{\left|x_{0}-y\right|^{m}}(1+|x-y| / t)^{-(\operatorname{delta}+(n+1) / 2)} d y \\
& \leq C \sum_{|\alpha|=m}|| D^{\alpha} A \|_{B M O} t^{-n} \sum_{k=0}^{\infty} \int_{2^{k+1} \tilde{Q} \backslash 2^{k} \tilde{Q}} \frac{\left|x-x_{0}\right||f(y)|}{\left|x_{0}-y\right|}(1+|x-y| / t)^{-(\delta+(n+1) / 2)} d y \\
& \leq C \sum_{|\alpha|=m}\left\|D^{\alpha} A\right\|_{B M O}\|f\|_{L^{\infty}(w)}, \\
& \left|L_{3}\right| \leq C \sum_{|\alpha|=m} t^{-n} \sum_{k=0}^{\infty} \int_{2^{k+1} \tilde{Q} \backslash 2^{k} \tilde{Q}}|f(y)|\left|D^{\alpha} \tilde{A}(y)\right|\left(1+\left|x_{0}-y\right| / t\right)^{-(\delta+(n+1) / 2)} d y \\
& \leq C \sum_{|\alpha|=m}(t / d)^{\delta-(n-1) / 2} \sum_{k=0}^{\infty} 2^{k((n-1) / 2-\delta)} \frac{1}{\left|2^{k+1} \tilde{Q}\right|} \int_{2^{k+1} \tilde{Q}}|f(y)|\left|D^{\alpha} A(y)-\left(D^{\alpha} A\right)_{\tilde{Q}}\right| d y \\
& \leq C \sum_{|\alpha|=m}\left\|D^{\alpha} A\right\|_{B M O}\|f\|_{L^{\infty}(w)} .
\end{aligned}
$$

Case 2. $t>d$. In this case, we choose $\delta_{0}$ such that $(n-1) / 2<\delta_{0}<$ $\min (\delta,(n+1) / 2)$, notice that (see [15])

$$
\left|B^{\delta}(x-y)-B^{\delta}\left(x_{0}-y\right)\right| \leq C\left|x-x_{0}\right|(1+|x-y|)^{-(\delta+(n+1) / 2)},
$$


similar to the proof of Case 1, we obtain

$$
\begin{aligned}
& \left|L_{1}\right| \leq C t^{-n} \int_{R^{n} \backslash \tilde{Q}} \frac{|f(y)|\left|R_{m}(\tilde{A} ; x, y)\right|}{\left|x_{0}-y\right|^{m+1}}\left|x_{0}-x\right|\left(1+\left|x_{0}-y\right| / t\right)^{-\left(\delta_{0}+(n+1) / 2\right)} d y \\
& +C t^{-n-1} \int_{R^{n} \backslash \tilde{Q}} \frac{|f(y)|\left|R_{m}(\tilde{A} ; x, y)\right|}{\left|x_{0}-y\right|^{m}}\left|x_{0}-x\right|\left(1+\left|x_{0}-y\right| / t\right)^{-\left(\delta_{0}+(n+1) / 2\right)} d y \\
& \leq C \sum_{|\alpha|=m}\left\|D^{\alpha} A\right\|_{B M O}(d / t)^{(n+1) / 2-\delta_{0}} \sum_{k=1}^{\infty} k 2^{k\left((n-1) / 2-\delta_{0}\right)}\|f\|_{L^{\infty}(w)} \\
& \leq C \sum_{|\alpha|=m}\left\|D^{\alpha} A\right\|_{B M O}\|f\|_{L^{\infty}(w)}, \\
& \left|L_{2}\right| \leq C t^{-n} \int_{R^{n} \backslash \tilde{Q}} \frac{|f(y)|\left|R_{m}(\tilde{A} ; x, y)-R_{m}\left(\tilde{A} ; x_{0}, y\right)\right|}{\left|x_{0}-y\right|^{m}}\left(1+\left|x_{0}-y\right| / t\right)^{-\left(\delta_{0}+(n+1) / 2\right)} d y \\
& \leq C \sum_{|\alpha|=m}\left\|D^{\alpha} A\right\|_{B M O}(d / t)^{(n+1) / 2-\delta_{0}} \sum_{k=1}^{\infty} 2^{k\left((n-1) / 2-\delta_{0}\right)}\|f\|_{L^{\infty}(w)} \\
& \leq C \sum_{|\alpha|=m}\left\|D^{\alpha} A\right\|_{B M O}\|f\|_{L^{\infty}(w)} \\
& \left|L_{3}\right| \leq C \sum_{|\alpha|=m}(d / t)^{(n+1) / 2-\delta_{0}} \sum_{k=0}^{\infty} 2^{k\left((n-1) / 2-\delta_{0}\right)} \frac{1}{\left|2^{k+1} \tilde{Q}\right|} \int_{2^{k+1} \tilde{Q}}|f(y)|\left|D^{\alpha} \tilde{A}(y)\right| d y \\
& \leq C \sum_{|\alpha|=m} \sum_{k=1}^{\infty} k 2^{k\left((n-1) / 2-\delta_{0}\right)} \frac{1}{\left|2^{k+1} \tilde{Q}\right|} \int_{2^{k+1} \tilde{Q}}|f(y)|\left|D^{\alpha} A(y)-\left(D^{\alpha} A\right)_{\tilde{Q}}\right| d y \\
& \leq C \sum_{|\alpha|=m}\left\|D^{\alpha} A\right\|_{B M O}\|f\|_{L^{\infty}(w)} .
\end{aligned}
$$

These yield the desired results. A same argument as in the proof of Theorem 1 will give the proof of (ii), (iii), (iv) and (v), we omit the details. This completes the proof of Theorem 3.

\section{ACKNOWLEDGEMENT}

The author would like to express his deep gratitude to the referee for his valuable comments and suggestions.

\section{REFERENCES}

1. Bui-Huy Qui, Weighted Hardy spaces, Math. Nachr., 103 (1981), 45-62.

2. S. Chanillo, A note on commutators, Indiana Univ. Math. J., 31 (1982), 7-16. 
3. W. G. Chen, G. E. Hu, Weak type $\left(H^{1}, L^{1}\right)$ estimate for multilinear singular integral operator, Adv. Math. (China), 30 (2001), 63-69.

4. J. Cohen, A sharp estimate for a multilinear singular integral on $R^{n}$, Indiana Univ. Math. J., 30 (1981), 693-702.

5. J. Cohen and J. Gosselin, On multilinear singular integral operators on $R^{n}$, Studia Math., 72 (1982), 199-223.

6. J. Cohen and J. Gosselin, A BMO estimate for multilinear singular integral operators, Illinois J. Math., 30 (1986), 445-465.

7. R. Coifman and Y. Meyer, Wavelets, Calderon-Zygmund and multilinear operators, Cambridge Studies in Advanced Math. Cambridge University Press, Cambridge, 1997.

8. R. Coifman, R. Rochberg and G. Weiss, Factorization theorems for Hardy spaces in several variables, Ann. of Math., 103 (1976), 611-635.

9. Y. Ding and S. Z. Lu, Weighted boundedness for a class rough multilinear operators, Acta Math. Sinica, 3 (2001), 517-526.

10. J. Garcia-Cuerva and J. L. Rubio de Francia, Weighted norm inequalities and related topics, North-Holland Math., Amsterdam, 1985.

11. E. Harboure, C. Segovia and J. L. Torrea, Boundedness of commutators of fractional and singular integrals for the extreme values of p, Illinois J. Math., 41 (1997), 676700 .

12. G. E. Hu and S. Z. Lu, The commutator of the Bochner-Riesz operator, Tohoku Math. J., 48 (1996), 259-266.

13. L. Z. Liu, Weighted weak type estimates for commutators of Littlewood-Paley operator, Japanese J. Math., 29(1) (2003), 1-13.

14. L. Z. Liu and S. Z. Lu, Weighted weak type inequalities for maximal commutators of Bochner-Riesz operator, Hokkaido Math. J., 32(1) (2003), 85-99.

15. S. Z. Lu, Four Lectures on Real $H^{p}$ Spaces, World Scientific, River Edge, NI, 1995.

16. E. M. Stein, Harmonic Analysis: Real Variable Methods, Orthogonality and Oscillatory Integrals, Princeton Univ. Press, Princeton NJ, 1993.

17. A. Torchinsky, The real variable methods in harmonic analysis, Pure and Applied Math., Academic Press, New York, 1986.

18. A. Torchinsky and S. Wang, A note on the Marcinkiewicz integral, Colloq. Math., 60/61 (1990), 235-243.

Liu Lanzhe

College of Mathematics and Computer,

Changsha University of Science and Technology,

Changsha 410077,

P. R. China

E-mail:lanzheliu@263.net 